\title{
Modelling of viscoelastic properties of a curing adhesive
}

\author{
J. de Vreugd ${ }^{1}$, K. M. B. Jansen ${ }^{1}$, L. J. Ernst ${ }^{1}$ \\ \& J. A. C. M. Pijnenburg ${ }^{2}$ \\ ${ }^{I}$ Delft University of Technology, Delft, The Netherlands \\ ${ }^{2}$ TNO Science and Industry, Delft, The Netherlands
}

\begin{abstract}
Thermoset adhesives are widely used in high tech applications to join two bodies together. The main advantages of using adhesives are the low weight of the construction and the easy way to apply the adhesive to the surfaces which have to be fixed together. The disadvantage of thermoset adhesives however is that cure shrinkage occurs. Shrinkage and evolution of mechanical properties during cure leads to development of internal stresses. In this paper, the mechanical behaviour of a curing adhesive is investigated. In the case of using a thermoset adhesive in high precision applications like optical instruments, care should be taken. Small displacements and distortions of important components caused by cure shrinkage may already lead to malfunctioning. For this reason a material model suitable for implementation in a finite element program is developed to predict stresses and strains in glued objects. The temperature and cure dependent viscoelastic shear modulus of the adhesive are obtained by using Dynamic Mechanical Analyzing methods. The bulk modulus is obtained at fully cured state with a high pressure dilatometer. Curing-time-time superposition is applied to model the shear modulus at any state of cure. It is assumed that the bulk modulus remains constant during cure. The kinetics of the adhesive is investigated by using Dynamic Scanning Colorimetric techniques. The relation between time and degree of cure is modelled by making use of the KamalSourour equation. Also diffusion limitation is added to this equation. The cure shrinkage of the adhesive is experimentally determined by making use of the principle of Archimedes. Finally a validation experiment is performed. The validation experiment is simulated in the finite element program ABAQUS and compared with the experiment. It turned out that the developed material model is accurate enough to predict reaction forces, stresses and strains caused by cure shrinkage.
\end{abstract}

Keywords: adhesive, cure shrinkage, DMA, DSC, viscoelastic properties. 


\section{Introduction}

Thermoset adhesives are used in many high-tech applications to fix two bodies together instead of other bonding techniques. Thermoset adhesives are often used when a low construction weight is required. Another advantage is the easy way of applying the glue to the surfaces. Next to above mentioned advantages of using an adhesive, also some negative properties are present. An important disadvantage of using an adhesive for bonding is the shrinkage of the adhesive during the transformation from a fluid to a solid material. The shrinkage results in distortions and internal stresses. In instruments where a high precision is required like optical instruments, cure shrinkage might cause problems. Displacements and rotations of important parts in high precision instruments are undesirable because of the necessary accurate position. It is even possible that cure shrinkage leads to cracks in a glued object. An example is shown in figure 1 where a glass plate is glued to a metal surrounding. In this example shrinkage forces were that high that the glass plate is cracked.

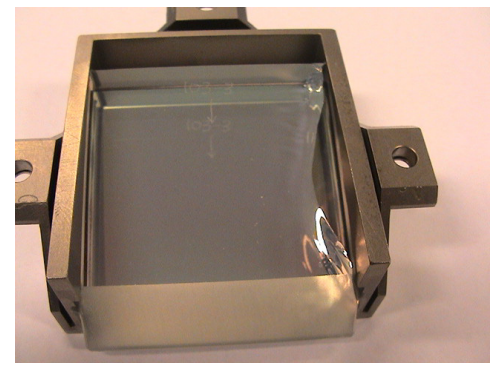

Figure 1: Cracks caused by cure shrinkage.

In order to avoid the mentioned problems, cure shrinkage should be taken into account at the design state of instruments where a high precision is required. To be able to produce a fail-proof design, a reliable model of both cure shrinkage and viscoelastic material properties is needed to predict stresses and strains during cure.

The Araldite AV $138 \mathrm{M}$ adhesive is a frequently used adhesive in aerospace. The cure shrinkage of this adhesive caused many problems in the past. For this reason is chosen to investigate the viscoelastic behaviour of this adhesive during the transformation from a fluid to a solid material.

The mechanical behaviour of the adhesive is completely characterized and modelled such that all viscoelastic properties are available at a large range in time, temperature and at a certain degree of conversion. The obtained model is used to solve the linear viscoelastic stress-strain relation:

$$
\sigma_{i j}(t)=2 \int_{-\infty}^{t} G(t-\xi) \dot{\varepsilon}_{i j} d \xi+\int_{-\infty}^{t}\left[K(t-\xi)-\frac{2}{3} G(t-\xi)\right]\left(\dot{\varepsilon}_{11}^{\text {eff }}+\dot{\varepsilon}_{22}^{\text {eff }}+\dot{\varepsilon}_{33}^{\text {eff }}\right) s_{i j}
$$

In equation (1) the variables $\mathrm{G}$ and $\mathrm{K}$ refer to shear and bulk modulus respectively, which are a function of time $(\mathrm{t})$, temperature $(\mathrm{T})$ and degree of 
conversion $(\alpha)$. The variable $\varepsilon_{i i}^{e f f}$ is the effective volumetric strain which includes cure shrinkage and thermal contributions. A model of both shear $\mathrm{G}(\mathrm{t}, \mathrm{T}, \alpha)$ and bulk $\mathrm{K}(\mathrm{T})$ modulus as well as cure shrinkage $\varepsilon_{\text {cure }}$ is proposed in this paper. The mechanical properties are experimentally found by using DMA (Dynamic Mechanical Analyzing) techniques and by using a high pressure dilatometer. The chemical reaction model is found by using DSC (Dynamic Scanning Calorimetric) measurements. Finally the decrease in density of the adhesive is measured during cure by using the method of Archimedes. All experimentally found properties are modelled so that they can be implemented in a finite element program.

In order to validate the material model of the adhesive during cure, a validation experiment was done. This experiment showed that the determined material model is accurate enough to do reliable predictions of stresses and strains.

\section{Cure kinetics}

Chemical reaction is started by applying a thermal loading to an uncured or not fully cured material. During this reaction, the individual epoxy monomers transform to a three dimensional network. This network prevents the molecules to slide past each other; this is the reason that a fluid like material transforms into a solid. The rate of reaction is dependent on the applied temperature. At the instant that the curing reaction is finished, one speaks about a fully cured material. The states between un- and fully cured situation are expressed by the expression: degree of cure or degree of conversion which is represented by the symbol $\alpha$. The value of $\alpha$ varies between 0 and 1 .

To be able to model the cure reaction it is necessary to describe the progress of the reaction, such that it is possible to calculate the degree of conversion at any moment of time and temperature. The reaction progress is measured in this research project by a DSC 2920 of TA instruments.

\subsection{Degree of cure determination}

During cure, heat comes free because of the chemical process (cross-linking). The degree of cure $\alpha$ is related to the maximum heat which comes free after a complete reaction $\left(\mathrm{H}_{\max }\right)$ and the heat which comes free after a certain state of reaction $(\mathrm{H})$. The degree of cure $(\alpha)$ is defined as $1-\mathrm{H} / \mathrm{H}_{\max }$. In order to measure the degree of conversion of a partly cured sample, it is necessary to calculate the total heat generated by a complete cure reaction.

A temperature ramp is applied to an uncured sample and the rate of heat generation $\mathrm{dH} / \mathrm{dt}$ is measured. Several heating rates $(\beta)$ are applied: $1,2,5$, $10^{\circ} \mathrm{C} / \mathrm{min}$. (dynamic scanning). The heat of reaction $\mathrm{H}$ is the amount of heat generated during dynamic scanning. The total generated heat, caused by the reaction is calculated for every measurement by the following equation: 


$$
H_{u}=\int_{0}^{t}\left(\frac{d H}{d t}\right) d t
$$

The thus obtained total heat of reaction varied between 130.27 and $135.42 \mathrm{~J} / \mathrm{g}$. A value of $135.42 \mathrm{~J} / \mathrm{g}$ is used in further calculations.

\subsection{Cure dependent $\mathbf{T}_{\mathrm{g}}$ determination}

Viscoelastic materials have the property that at low temperatures the material behaves glassy and at high temperatures more rubbery. The temperature at which this behaviour changes from a glassy to a rubbery behaviour is the glass transition temperature $\left(T_{g}\right)$. The glass transition temperature is a cure dependent property and is therefore measured as a function of degree of conversion. This property is measured as a sudden change in heat capacity $\mathrm{C}_{\mathrm{p}}$ (Seifi et al [1]). The heat capacity is measured by applying DSC scans to samples of different conversion levels. The results of these tests are shown in figure 2.

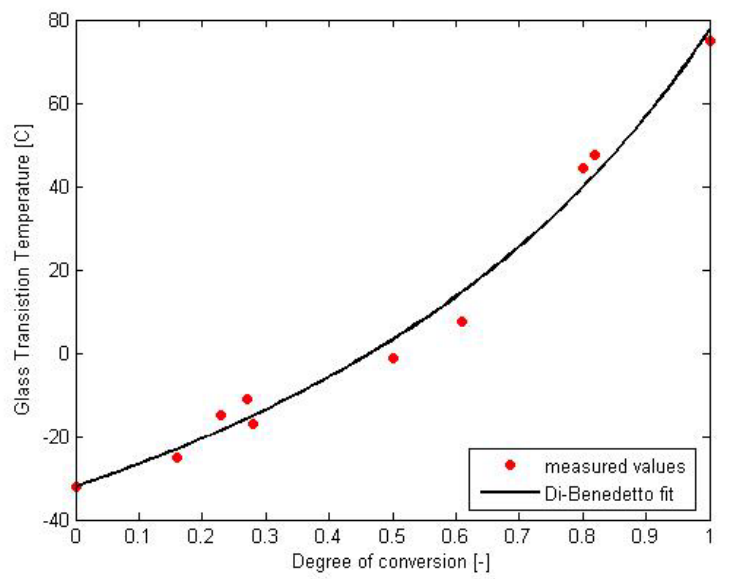

Figure 2: Glass transition temperature as a function of degree of conversion.

The above measured data points are fitted to the Di-Benedetto equation:

$$
T_{g}(\alpha)=T_{g 0}+\frac{\left(T_{g f}-T_{g 0}\right) \cdot \lambda \cdot \alpha}{1-(1-\lambda) \cdot \alpha}
$$

$\mathrm{T}_{\mathrm{gf}}$ and $\mathrm{T}_{\mathrm{g} 0}$ represent $\mathrm{T}_{\mathrm{g}}$ at fully and uncured state respectively. The measurements showed that, $\mathrm{T}_{\mathrm{gf}}=77.5^{\circ} \mathrm{C}$ and $\mathrm{T}_{\mathrm{g} 0}=-32.1^{\circ} \mathrm{C}$. The value $\lambda$ is a material dependent parameter. For the adhesive studied in this paper, $\lambda=0.474$.

\subsection{Kinetic model}

The chemical reaction is described by the model of Kamal and Sourour. This model is given in equation (4). 


$$
\frac{d \alpha}{d t}=k_{0} e^{\left(\frac{-E_{a}}{R T}\right)} \cdot \alpha^{m}(1-\alpha)
$$

In this equation $\mathrm{E}_{\mathrm{a}}$ denotes an activation energy (Starink [2]), $\mathrm{R}$ denotes the universal gas constant $\approx 8.314 \mathrm{~J} /(\mathrm{mol} \cdot \mathrm{K})$ and $\mathrm{T}$ represents the absolute temperature as a function of time. The parameters $\mathrm{k}_{0}, \mathrm{~m}$ and $\mathrm{n}$ are fit variables. The following values are found: $\mathrm{k}_{0}=3.2982 \cdot 10^{5}, \mathrm{~m}=0.185, \mathrm{n}=1.5154$

During isothermal curing, a thermosetting resin vitrifies if the reaction temperature is lower than the maximum glass transition temperature of the fully cured material. Due to the vitrification process, the kinetics becomes diffusion controlled. This phenomenon is also observed in the studied adhesive. It turned out that a sample cured at a room temperature could not reach maximum conversion. The maximum conversion level turned out to be $81 \%$. Therefore, the kinetics model is modified to:

$$
\frac{d \alpha}{d t}=\left[\frac{d \alpha}{d t}\right]_{\text {chem }} \cdot f_{d}
$$

[da/dt] describes the chemically controlled kinetics. Kamal-Sourour's equation is used here. $f_{d}$ denotes the diffusion control function (Schawe [3]). If the reaction is chemical controlled $f_{d}$ is equal to unity. In case of diffusion controlled reaction $f_{d}$ will have a value between 0 and 1 . The diffusion control function has to show an inflection point if the glass transition temperature is equal to the reaction temperature. A model for this function has to describe the inflection point properly. The following equation is fulfilling the mentioned requirements:

$$
f_{d}(\alpha)=1-\left(1+\frac{1}{2}\left(\frac{T_{\text {react }}+\Delta T-T_{g}(\alpha)}{\Delta T}\right)^{3}\right)^{-1}
$$

$\mathrm{T}_{\text {react }}$ is the temperature where the reaction place. $\Delta \mathrm{T}$ is a fitting parameter; for this adhesive is found by trial and error that $\Delta \mathrm{T}$ is $21.5^{\circ} \mathrm{C}$.

\section{Mechanical properties}

To be able to predict the stresses in a glued object, it is necessary to know the mechanical properties of the adhesive. The properties have to be known at a fully cured state, as well as during the curing trajectory. For the viscoelastic elongation- and shear-modulus a Dynamic Mechanical Analyzer (DMA) is used. The used test device for these measurements is a DMA Q800 of TA-instruments. This instrument has a displacement resolution of $1 \mathrm{~nm}$ and a force resolution of $1 \mathrm{mN}$. The bulk modulus is measured by a high pressure dilatometer. A GNOMIX dilatometer with a pressure range of $200 \mathrm{MPa}$ is used.

\subsection{Tensile modulus of fully cured adhesive}

In order to measure the viscoelastic properties of the fully cured material, a test bar was required. The used dimensions are [22.89 x $3.1 \times 0.82 \mathrm{~mm}]$. The test bar 
is produced by curing the adhesive in a suitable mold. Before curing the material submitted to vacuum to subtract the gas bubbles in the uncured resin. A cure temperature of $75^{\circ} \mathrm{C}$ is applied for 5 hours.

The test bar is exposed to a sinusoidal strain with different frequencies: 0.3 , $0.65,1.4,3,6.5,13.8,30,64.6,130 \mathrm{~Hz}$. During the frequency sweeps a temperature ramp is applied from $-50^{\circ} \mathrm{C}$ to $220^{\circ} \mathrm{C}$ with a heating rate of $1^{\circ} \mathrm{C} / \mathrm{min}$. The result of this experiment is shown in figure 3 .

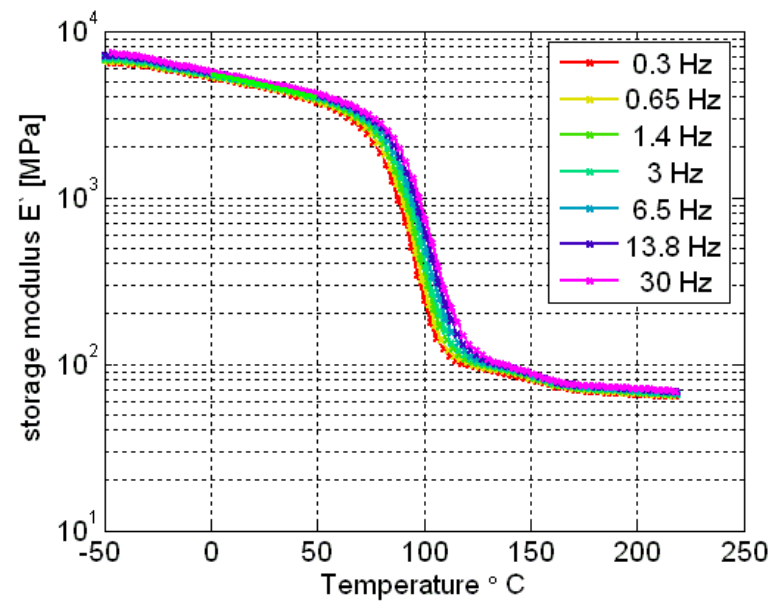

Figure 3: $\quad$ Result of DMA experiment to a fully cured bar of adhesive.

From this figure it is concluded that the glass-plateau of this material is:

$$
E_{\text {glass }}=5517 \cdot 10^{6}-30.452 \cdot 10^{6} \cdot T[\mathrm{MPa}]
$$

By applying the time-temperature superposition principle (Nielsen and Landel [4]) to the rough data, a mastercurve is obtained. The shiftfactors $\left(a_{t}\right)$ are fitted to the Williams-Landel-Ferry equation:

$$
\log \left(a_{t}\right)=\frac{C_{1}\left(T-T_{R}\right)}{C_{2}+T-T_{R}}
$$

The constants are treated as fit variables; $\mathrm{T}_{\mathrm{g}}$ is taken as the reference temperature $\mathrm{T}_{\mathrm{R}}$. By applying a non-linear fit, it is found that $\mathrm{C}_{1}=1.51, \mathrm{C}_{2}=28.6 . \mathrm{T}_{\mathrm{g}}$ is the value where $\tan (\delta)$ at $1 \mathrm{~Hz}$ reaches a maximum. $\mathrm{T}_{\mathrm{g}}$ turned out to be $84^{\circ} \mathrm{C}$.

\subsection{Bulk modulus measurement}

A GNOMIX high pressure dilatometer is used to measure the bulk modulus. A sample with a typical mass of 1.5 gram is contained in a rigid cell, closed by flexible bellows. This cell contains mercury to fill the cell completely. The cell is placed into a vessel which can be heated. A hydrostatic pressure applied to the cell, will result in a deflection of the bellows. This deflection can be related to deformations inside the cell. The pressure range that can be applied to the sample varies between 10 and $200 \mathrm{MPa}$, the highest applicable temperature is $400^{\circ} \mathrm{C}$. 
In order to measure the bulk modulus of the material, a stepwise temperature scan is applied to the material. At every temperature step, steps of 10MPa are applied. Equation (9) (Fung [5]) is used to calculate the bulk modulus:

$$
K=\Delta p \frac{-v}{\Delta v}
$$

The result of this measurement is presented in figure 4 .

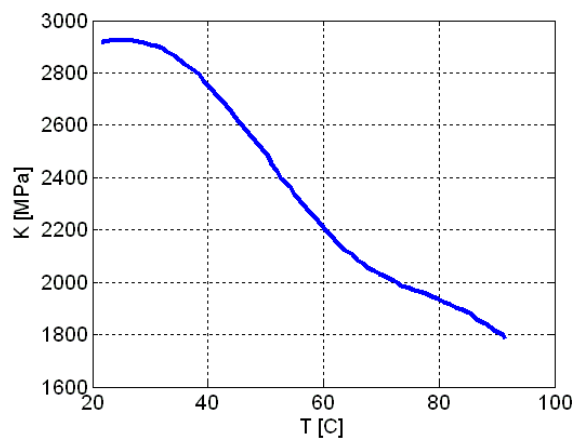

Figure 4: Bulk modulus as a function of temperature.

In figure 4 is shown that the maximum modulus value is about $2900 \mathrm{MPa}$, the lowest about $1800 \mathrm{MPa}$. For temperatures above $50^{\circ} \mathrm{C}$ the material is time dependent so for these temperatures the measured values cannot be used for finite element simulations but it gives an estimate of the modulus at those particular temperatures.

\subsection{Shear modulus during cure}

To be able to predict stresses and strains in a glued object during cure, it is necessary to know the viscoelastic properties during cure. The cure dependent shear modulus is determined by measuring the change in stiffness of a droplet of adhesive which is clamped between 2 plates. To one of the plates a sinusoidal strain of $5 \mu \mathrm{m}$ is applied. By recording the forces and amplitudes of the plate during the experiment, the stiffness $\mathrm{K}$ of the sample is calculated. With the known dimensions of the droplet of adhesive the shear modulus is calculated:

$$
G(\omega)=K(\omega) \cdot\left(\frac{2 h}{A}\right)
$$

In equation (10), $\mathrm{h}$ and $\mathrm{A}$ represents the gap between the plates and the crosssectional surface of the adhesive sample respectively. Different isothermal loadings are applied to sample such that the adhesive cures during the experiment. Three experiments are done with isothermal loadings of $40^{\circ} \mathrm{C}, 45^{\circ} \mathrm{C}$ and $50^{\circ} \mathrm{C}$. The results of these experiments are plotted in figure 5 .

In figure 5 the viscoelastic shear modulus is plotted as a function of degree of conversion. At conversion levels lower than 0.55 , the material is still a fluid. The shear modulus in this region is 0 . In order to obtain a mastercurve of the shear- 
modulus, the cure-time-time superposition principle (Yongsung [6]) is used. The shiftfactors which are used for determining the mastercurves are fitted to the following equation:

$$
\operatorname{Shift}(\alpha, T)=10^{C_{1}+C_{2} \cdot T+C_{3} \cdot e^{\left(C_{4} \cdot \alpha\right)}}
$$

The following values for the fit factors are found: $C_{1}=-2.361, C_{2}=-0.150$, $\mathrm{C}_{3}=16.17, \mathrm{C}_{4}=-3.66$.
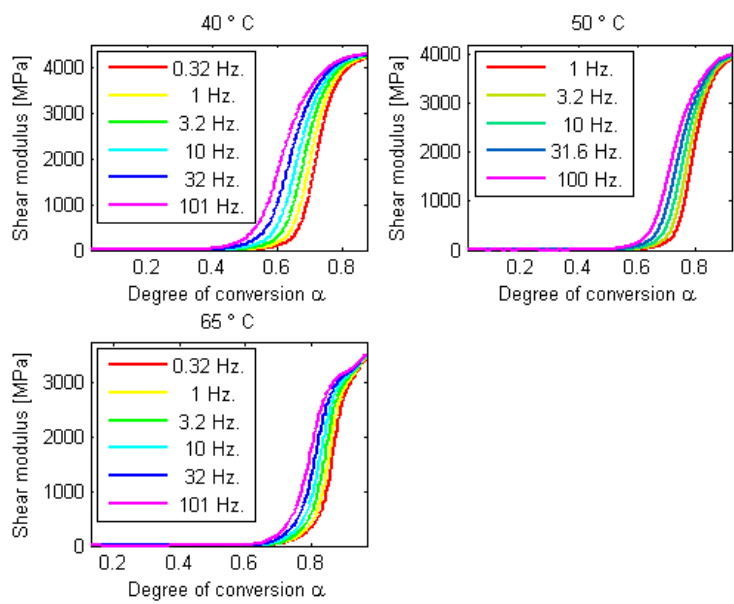

Figure 5: Result of shear tests during cure.

\section{Cure shrinkage}

The cure shrinkage is experimentally found by making use of Archimedes' principle. An apparatus is designed which makes use of buoyancy forces caused by immersing a body in a fluid. By knowing the mass of the sample, the weight of the mass immersed in the fluid, and the density of the fluid, the density of the sample can be calculated. The mass of the sample and the density of the fluid should be known before doing the measurement. As an immersing fluid, silicone oil is used with a density of $0.9670 \mathrm{~g} / \mathrm{cm}^{2}$ and a CTE of $8.20 \cdot 10^{-4} / \mathrm{K}$. Different isothermal loadings are applied to samples: $20^{\circ} \mathrm{C}$ for $70 \mathrm{hrs}, 40^{\circ} \mathrm{C}$ for $18 \mathrm{hrs}$ and $50^{\circ} \mathrm{C}$ for $16 \mathrm{hrs}$. The result of the measurement at $20^{\circ} \mathrm{C}$ is shown in figure 6 .

From figure 6 is concluded that there is linear relation between degree of cure and density. The total volumetric cure shrinkage $\gamma_{\mathrm{v}}$ is calculated with the following equation:

$$
\gamma_{v}=\frac{\Delta v}{v_{f u l l y}}=\frac{-\Delta \rho}{3 \cdot \rho_{f u l}}=\frac{0.0704}{3 \cdot 1.7674}=0.0133
$$




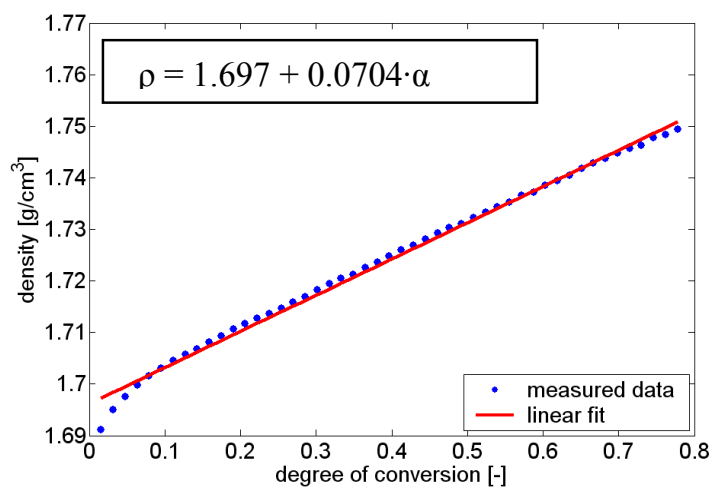

Figure 6: Results of density measurement.

\section{Validation experiment}

In order to check the accuracy of the obtained material model, a validation experiment is done, see Figure 7. In this experiment a droplet of adhesive is applied in the middle of a glass-plate which is fixed at both ends. Dimensions of the glass-plate were: [ $40 \times 10 \times 2 \mathrm{~mm}$ ].

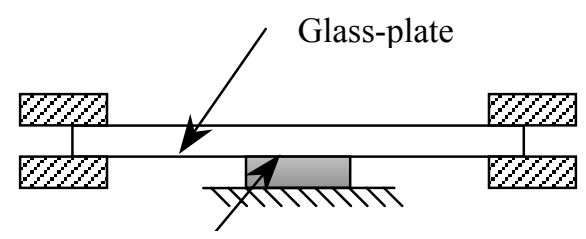

Adhesive bump

Figure 7: Schematic drawing of validation experiment.

Due to the shrinkage of the adhesive, the glass-plate will deflect. A temperature load is applied to the adhesive, firstly a temperature of $40^{\circ} \mathrm{C}$ is applied for 35 hours, after that the temperature is changed to $80^{\circ} \mathrm{C}$ for 20 hours. In the validation experiment, the reaction force at the bottom of the adhesive bump is measured. Simultaneously the force is calculated by using the finite element program ABAQUS. User-subroutines were used to implement the obtained material model. A picture of the used mesh is given in figure 8 .

Shell-elements are used for the glass-plate, and solid elements are used for the adhesive bump. Measured and calculated forces are presented in figure 15.

In figure 9, the calculated forces are compared to the measured forces. The calculated forces are about $30 \%$ too high. This is probably due to the bulk modulus which was implemented in the simulation model as a non-time and noncure dependent value. Another reason is most probably due to friction at the boundary conditions. This is not modelled due to a lack of time. 


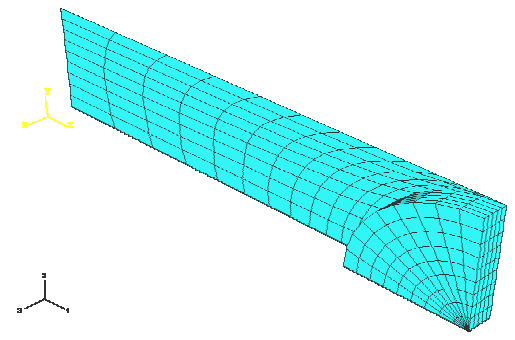

Figure 8: $\quad$ Mesh of the validation experiment.
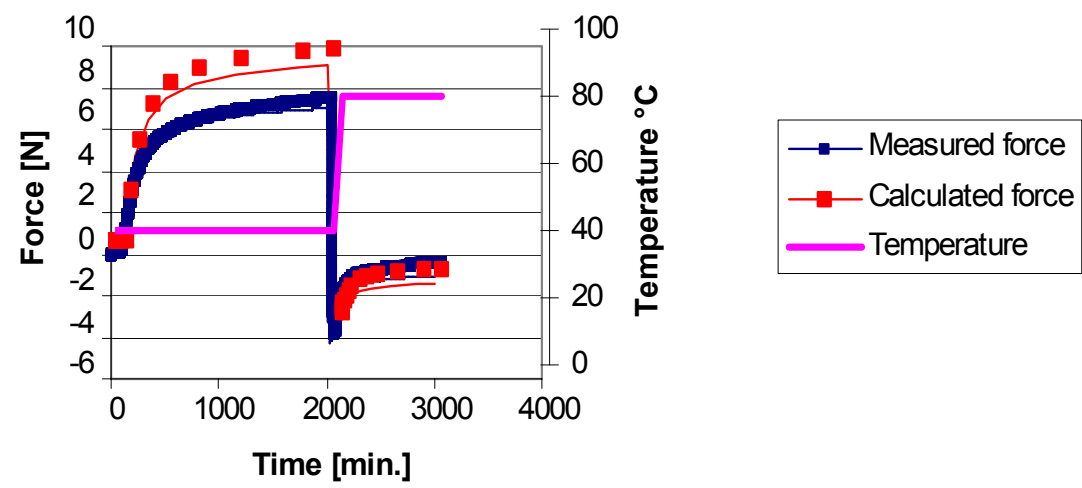

Figure 9: Result of validation experiment.

\section{Conclusions and recommendations}

In this research, a first start is made in characterising the mechanical properties of the adhesive Araldite AV 138M. The mechanical properties which are a function of time, temperature and degree of conversion are studied and fitted in a material model. The kinetics of this material is well described in a relation in which also diffusion limitation is implemented. The cure shrinkage is found and modelled. The material model is implemented in ABAQUS.

The following properties of the investigated adhesive were established during this work:

- $\mathrm{T}_{\mathrm{g}}$ varies between $-32^{\circ} \mathrm{C}$ and $77.5^{\circ} \mathrm{C}$ during cure. The relation between $\mathrm{T}_{\mathrm{g}}$ and degree of conversion is well described by Di-Benedetto's equation.

- The kinetics is modelled by making use of Kamal-Sourours' equation. Diffusion limitation is added to this model.

- The fully cured elongation modulus varies between $6500 \mathrm{MPa}$ at $-50^{\circ} \mathrm{C}$ and $65 \mathrm{MPa}$ at $200^{\circ} \mathrm{C}$.

- The bulk modulus varies between $2700 \mathrm{MPa}$ at $30^{\circ} \mathrm{C}$ to $1800 \mathrm{MPa}$ at $90^{\circ} \mathrm{C}$.

- A simple cure and temperature dependent shiftfactor is obtained, with which mastercurves at other conversion levels can be found. 
- The decrease in density is about $4 \%$. It turned out that there is a linear relationship between degree of conversion and density.

- From the validation experiment is concluded that the obtained material model is accurate enough for predicting stresses and strains in glued objects.

For future work some recommendations are listed below:

- More validation experiments should be done. Some parameters of the validation experiment can be changed. For instance the thickness of the adhesive layer. It might be that the reaction forces caused by cure shrinkage are very sensitive to the applied layer thickness.

- It turned out that there is an error of about $30 \%$ between the measured and simulated reaction forces. This is probably caused by an inaccurate bulk modulus. So, bulk modulus has to be found as a function of time, temperature and degree of conversion.

\section{References}

[1] Seifi, R., Hojjati, M., Heat of reaction, cure kinetics and viscosity of araldite LY-556 resin, Journal of composite materials, 39(11), pp. 10271039, 2005.

[2] Starink, M.J., The determination of activation energy from linear heating rate experiments: a comparison of the accuracy of isoconversion methods, Thermochimica acta, 404(1-2), pp. 163-176, 2003.

[3] Schawe, J.E.K., A description of chemical and diffusion control in isothermal kinetics of cure kinetics, Thermochimca Acta 388(1-2) pp. 299312, 2002.

[4] Nielsen, L.E., Landel, R.F., Mechanical properties of polymers and composites, pp.110, 1988.

[5] Fung, Y.C., Foundations of solid mechanics, pp. 113, 1984.

[6] Yongsung E.O.M., Louis Boogh et al., Time-cure-temperature superposition for the prediction of instantaneous viscoelastic properties during cure, Polymer engineering and science, 60(6), pp. 521-528, 2002. 